\title{
Effect of impact energy on the shape of granular heaps
}

\author{
Yan Grasselli ${ }^{1,2}$, Hans J. Herrmann ${ }^{1,2}$, Gadi Oron ${ }^{2}$ and Stefano Zapperi ${ }^{2}$ \\ ${ }^{1}$ ICA 1, University of Stuttgart, Pfaffenwaldring 27, 70569 Stuttgart, Germany \\ ${ }^{2}$ PMMH-ESPCI, 10 Rue Vauquelin, 75231 Paris CEDEX 05, France.
}

September 4, 2018

\begin{abstract}
We study experimentally the shape of a granular heap formed pouring a granular material into a vertical Hele-Shaw cell and analyze the effect of the grain impact energy. We propose a continuous model for the steady profile of the heap that explicitly considers energy dissipation of flowing grains through inelastic collisions. We solve the model analytically and analyze the resulting height profile as a function of several parameters, such as the restitution coefficient of the grains and their impact energies. We find good agreement between theory and experiments.
\end{abstract}

\section{Introduction}

The study of granular materials has received wide attention in the last few years [1, 2, 33, because of the challenging problems posed by this state of matter. Sand heaps are among the most familiar granular systems that have been studied experimentally and theoretically. Despite the apparent simplicity a sand heap presents a number of intriguing phenomena, such as avalanches [4] internal texture [5], segregation and stratification [6]. Several theoretical models have been employed to describe the dynamics and the static profile of a sandpile, ranging from purely numerical models, such as lattice gas [7, 8] and molecular dynamics simulations [9], to discrete lattice models [10] and continuous equations [1], 12].

Granular heaps can be generated by slowly pouring grains (i.e. spherical beads, sand) from a given height over a rigid support (3D piles) or into a vertical Hele-Shaw cell (2D piles). The pile typically displays a well defined slope, corresponding to the angle of repose, with clear deviations at the top and at the tail. In particular, the tail of a $2 \mathrm{D}$ pile was found experimentally to follow a logarithmic dependence [13, 14]. This result was anticipated by the solution of a continuous model solved in a 2D silo geometry [12] and by theoretical arguments 13. The angle of repose depends on the characteristics of the material such as density, humidity, packing history and boundary conditions. 
Here we analyze the shape of the top of the granular heap, focusing on the effect of the impact energy of the particles. To this end, we perform a set of experiments with glass and leads beads poured at very slow rate into a vertical Hele-Shaw cell and study the changes of the heap profile when grains are poured from different heights. We propose a simple continuous model to describe the steady-state properties of the grains flowing on the top of the pile, providing an explicit expression for the heap profile. The model treats explicitly the dissipation of the kinetic energy of the flowing grains due to inelastic collisions and provides a good fit to the experimental data on glass and lead beads.

\section{Experiments}

Experiments are performed within a vertical Hele Shaw cell $(20 x 30 \mathrm{~cm})$ with a fixed thickness of $5 \mathrm{~mm}$ [15. Lead beads, with diameter $d=0.2 \mathrm{~cm}$ are poured through a funnel placed above the center of the cell. We carefully determine the impact energy of the particles, controlling the distance $Z_{0}$ between the top of the heap and the funnel: the impact energy is thus $e_{0}=m g Z_{0}$. The falling heights range from $Z_{0}=1 \mathrm{~cm}$ to $Z_{0}=14 \mathrm{~cm}$ and are determined with a with a precision of $\Delta Z_{0}=0.5 \mathrm{~cm}$. Experiments are performed at very low particle flux, dropping one particle at a time through the funnel. In the range of falling heights employed [16], the reorganization of the pile after impact is usually very weak and the particle jumps down the slope and is eventually trapped, giving rise to a local change in the slope of the pile. The dynamics of the beads can be sometimes very complex: we observe collisions with the walls of the cell, rolling motion, ejection of other particles and occasionally big avalanches of a few layers.

After a short transient stage, the heap grows steadily and the profile translates at constant velocity, proportional to the input flux. In this regime we retrieve the experimental heap profiles (see Fig. 11)from image analysis and we average them. The averaging is done superimposing the profiles obtained at different time steps in the steady state. In Fig. 2 we show the averaged profiles for different impact energies. It can be observed that the heap has a flat top and shows a well defined angle of repose, which turns out to be essentially independent on $Z_{0}$, at least for the experimental values used. When the tails of the heaps obtained with different $Z_{0}$ are superimposed, we observe that the maximum height $h(0)$ decreases linearly with $Z_{0}$ (inset of Fig. 2). We find that the shape of the heap is well described by the form

$$
h(x)=A-\theta_{c} x-\theta_{c} \xi e^{(-x / \xi)},
$$

where $\theta_{c}$ is the angle of repose, $\xi$ is a correlation length and $A$ is an inessential constant, that can be eliminated through an appropriate vertical translation of the profile. From Eq. (1) we have that $h(0)=A-\theta_{c} \xi$, which implies that $\xi$ is proportional to $Z_{0}$, which is verified by the fitting parameters (see Fig. 3) This result reflects the fact that $Z_{0}$ is, together with the particle diameter, the only characteristic length present in the system. 
The basic features of these results are quite robust and do not depend on the particular material used. We verified this by forming heaps with glass beads of considerably smaller diameter $(d=0.025 \mathrm{~cm})$, poured at low flux $W=0.32 \mathrm{~g} / \mathrm{s}$. In this case $Z_{0}$ can be controlled very precisely by moving the outlet up at the same velocity as the growth of the top of the heap. In this experiment, grains are poured from one of the two sides of the Hele-Shaw cell. We see in Fig. 1 that also in this case $h(0)$ is linear in $Z_{0}$ and the decay is reasonably well fitted by an exponential, excluding from the fitting region the first $0.5 \mathrm{~cm}$, which correspond to the radius of the funnel. Finally in this case, the measured angle of repose displays a small dependence on $Z_{0}$, of the order of $\Delta \theta=0.05$. Looking at Fig. [3, we note that the linear dependence of $\xi$ from $Z_{0}$ is steeper in the case of glass beads by roughly a factor 2 . The origin of this effect is probably due to the different restitution coefficients of the materials.

\section{Model}

In order to understand the experimental observations, we consider a sandpile model in which energy dissipation is explicitly treated. The complete dynamical evolution of all the grains and their energy dissipation is extremely difficult to treat explicitly. The evolution of the heap is indeed a very complex phenomenon, being due to the way momentum is transfered during particle collisions, frictional properties and other forms of energy dissipation. However, we note that in our experiment the growth of the heap displays a remarkable stationary state, which we can try to describe with a simplified model taking into account essentially only energy dissipation.

Alonso et al. 10] have introduced a simple automaton model which has been used to derive the angle of repose of a pile in terms of microscopic parameters, such as the restitution coefficient of the grains. In this model, grains are poured one by one on the top of a heap with an initial kinetic energy $e_{0}$. As the grains fall down the pile, parameterized by the local height $h(x)$, their kinetic energy $e$ decreases due to inelastic collisions and increases because of gravity as

$$
e(x+\delta)=r(e(x)+m g(h(x)-h(x+\delta))),
$$

where $m$ is the mass of the grains, $\delta$ is the characteristic size of the jumps, $g$ is the gravity acceleration and $r$ is an "effective" restitution coefficient. The grains jump to the right until their kinetic energy is less than a threshold $u$. When a grain comes to rest at $x$ the height $h$ of the column $x$ is increased by one unit and a new grain is poured at the origin. This model has been simulated and, despite its simplicity, was shown to explain several experimental results [17].

We study here a continuous version of the model in which the steady-state kinetic energy of the rolling grains follows Eq. (2)

$$
\delta \frac{d e(x)}{d x}=(r-1) e(x)+r \gamma(x),
$$

where $\gamma(x) \equiv-m g \delta d h / d x$ measures the local slope. Eq. (3) describes the steady-state energy profile in terms of a given slope profile $\gamma(x)$. To close the 
problem, we need to specify how the steady-state slope profile depends on the energy profile. We assume that the system in the steady state is at the verge of stability and that the variations of the local slope compensate the difference between the kinetic energy $e$ and the trapping energy $u$ : the slope will decrease for $e>u$ and increase for $e<u$. The simplest equation of this form is given by

$$
\delta \frac{d \gamma(x)}{d x}=\Gamma(u-e(x))
$$

where $\Gamma$ is a phenomenological parameter. Eq. (4) implies that for large $x$ the grains are at the threshold of mobility $e(x) \simeq u$.

Finally, we impose the boundary conditions $e(0)=e_{0}=m g Z_{0}$ and $\gamma(0)=0$. We note that by dividing each term of the equation by $m g$ all the quantities can be expressed in terms of lengths. Eqs. (3-4) are linear and can be solved in order to obtain an explicit expression for the profile of the pile. It is convenient to transform the pair of equations into an homogeneous one using the transformation

$$
E(x)=e(x)-u, \quad G(x)=\gamma(x)+(1-r) u / r .
$$

The solution of the homogeneous equations

$$
\begin{array}{r}
\delta \frac{d e(x)}{d x}=(r-1) E(x)+r G(x), \\
\delta \frac{d G(x)}{d x}=-\Gamma E(x),
\end{array}
$$

is given by

$$
\begin{array}{r}
e(x)=u+E_{+} e^{\lambda_{+} x / \delta}+E_{-} e^{\lambda_{-} x / \delta} \\
\gamma(x)=(r-1) u / r+G_{+} e^{\lambda_{+} x / \delta}+G_{-} e^{\lambda_{-} x / \delta}
\end{array}
$$

where $\lambda_{ \pm}=\left(r-1 \pm \sqrt{(r-1)^{2}-4 r \Gamma}\right) / 2$ are the eigenvalues of the matrix

$$
\mathcal{L} \equiv\left(\begin{array}{cc}
r-1 & r \\
-\Gamma & 0
\end{array}\right)
$$

The coefficients $E_{ \pm}$and $G_{ \pm}$can be explicitly obtained imposing the boundary conditions.

In the limit of large $x$ the slope of the pile is given by $\gamma_{\infty}=(r-1) u / r$, from which we obtain the angle of repose $\theta_{c}=(1-r) u /(m g \delta r)$. Since the real part of $\lambda_{ \pm}$is negative for all the values of the parameters the model has a well defined angle of repose. The relaxation towards the angle of repose is expressed by the sum of two exponentials with characteristic lengths $\xi_{ \pm} \equiv \delta / \lambda_{ \pm}$. Since $\xi_{+}>\xi_{-}$, the relaxation is dominated by $\xi_{+}$that we can identify with $\xi$ of Eq. 1. Finally, integrating the equation for $\gamma(x)$, we obtain an analytical form for the shape of the pile that can be compared with experiments

$$
h(x)=h(0)-(1-r) u x /(m g \delta r)-
$$




$$
\delta\left[G_{+} / \lambda_{+}\left(e^{\lambda_{+} x / \delta}-1\right)+G_{-} \lambda_{-}\left(e^{\lambda_{-} x / \delta}-1\right)\right] .
$$

The exponential relaxation to the angle of repose is experimentally observed, although in the present form the model can not account for the impact energy dependence of the relaxation length.

An original assumption of Eq. (2) was that the jump length $\delta$ is constant during the grain motion. The problem of a ball rolling and jumping over a rough inclined plane has received a large attention in recent years 18, 19. It has been experimentally observed that as a function of the angle of the inclined plane the motion is decelerated or accelerated, with a region in between where the motion is effectively viscous. The energy relaxation was recently studied in Ref. 19], where the stopping length in the decelerated regime was measured as a function of the initial energy. The result clearly indicates that the stopping length is linearly dependent on the initial energy. We can show this fact by a simple argument. Consider an inelastic ball hitting a plane with energy $e_{0}$ and let $\alpha$ be the angle between the plane and the particle velocity before the impact. We consider for simplicity that normal and tangential restitution coefficients are both equal to $r$ so that at each jump $n$ the energy decreases by $e_{n}=r e_{n-1}$. A straightforward calculation shows that the jump length decreases with $n$ as $\delta_{n}=2 e_{0} r^{n} \sin (2 \alpha) /(m g)$, which yields $e(x)=e_{0}\left[1-m g x(1-r) /\left(2 e_{0} \sin (2 \alpha)\right)\right]=$ $e_{0}(1-x / \xi)$. We see here that the characteristic length for energy relaxation $\xi$ is linear in $e_{0}$, in agreement with the experimental result seen in Fig. 3

\section{Conclusions}

In conclusion, we have studied the effect of impact energies on the shape of the sand heap. Experiments show an exponential relaxation of the slope to the angle

of repose, with a characteristic length that is linearly dependent on the impact energy of the grains. We model this process by simple relaxation equations and explain theoretically the experimental results.

S. Z. is supported by EC TMR Research Network contract ERBFMRXCT960062.

\section{References}

[1] H. J. Herrmann, J. P. Hovi and S. Luding (eds), Physics of dry granular media (Kluver, Dordrecht, 1998).

[2] H. M. Jaeger and S. R. Nagel, Science 255, (1992) 1523.

[3] A. Hansen and D. Bideau (eds.), Disorder and Granular Media (North Holland, Amsterdam, 1992).

[4] H. M. Jaeger, C. H.- Liu and S. R. Nagel, Phys. Rev. Lett. 62, (1989) 40.

[5] M. E. Cates, J. P. Wittmer, J.-P. Bouchaud, and P. Claudin, Phys. Rev. Lett 81, (1998)1841. 
[6] H. A. Makse, S. Havlin, P. R. King and H. E. Stanley, Nature 386, (1997) 379 ; H. A. Makse, P. Cizeau and H. E. Stanley, Phys. Rev. Lett. 78, (1997) 3298 .

[7] A. Károlyi, and J. Kertész, Phys. Rev. E 57, (1998) 852.

[8] G. Peng and H. J. Herrmann, Phys. Rev. E 48, (1994) R1796.

[9] S. Luding and H. -G. Matuttis, in Friction, Arching and Contact Dynamics, edited by D. E. Wolf and P. Grassberger (World Scientific, Singapore, 1997).

[10] J. J. Alonso, J.-P. Hovi and H. J. Herrmann, Phys. Rev. E 58, (1998) 672.

[11] J. P. Bouchaud, M. Cates, R. Prakash and S. F. Edwards S.F., Phys. Rev. Lett. 74, (1995) 1982.

[12] T. Boutreux and P.-G. de Gennes, J. Phys. I 6, (1996) 1295.

[13] J. J. Alonso and H. J. Herrmann, Phys. Rev. Lett. 76, (1996) 4911.

[14] Y. Grasselli and H. J. Herrmann, C. R. Acad. Sci. (Paris) 326, (1998) 61.

[15] The tail of the heap is affected by the presence of a confining geometry (i.e. silos) [12, 14]. This is not the case for the top of the heap, which we are studying here.

[16] We are currently performing experiments with large impact energies, with $Z_{0}$ of the order of a few meters. In this case the behavior is completely different from the one studied here: we observe a large reorganization of the pile after each impact and the formation of a crater at the top of the pile.

[17] M. Rimmele, M. Sc. thesis, University of Stuttgart, 1997 (in German).

[18] G. G. Batrouni, S. Dippel and L. Samson, Phys. Rev. E 53, (1996) 6496; S. Dippel, G. G. Batrouni and D. Wolf, ibid. 54, (1996) 6845; ibid. 56, (1997) 3645 .

[19] C. Henrique, M. A. Aguirre, A. Calvo, I. Ippolito, S. Dippel, G. G. Batrouni and D. Bideau, Phys. Rev. E 57, (1998) 4743. 


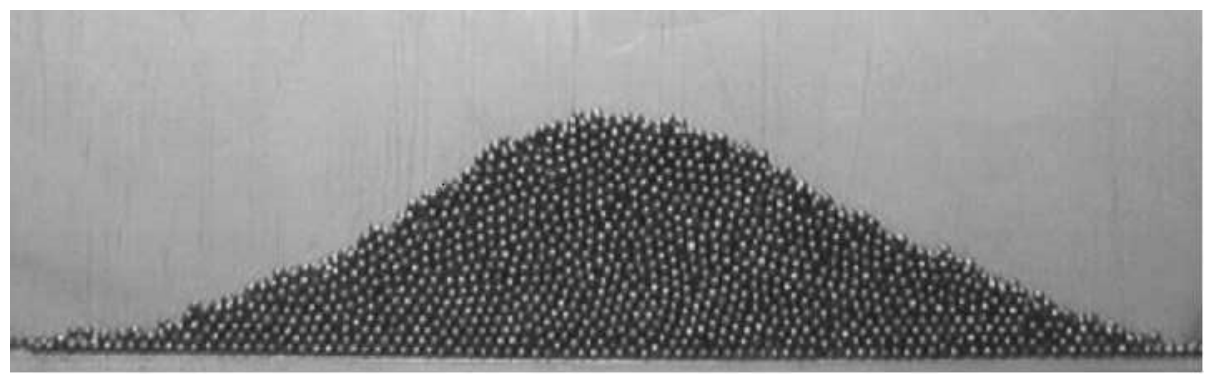

Figure 1: An example of a heap obtained experimentally with lead beads.

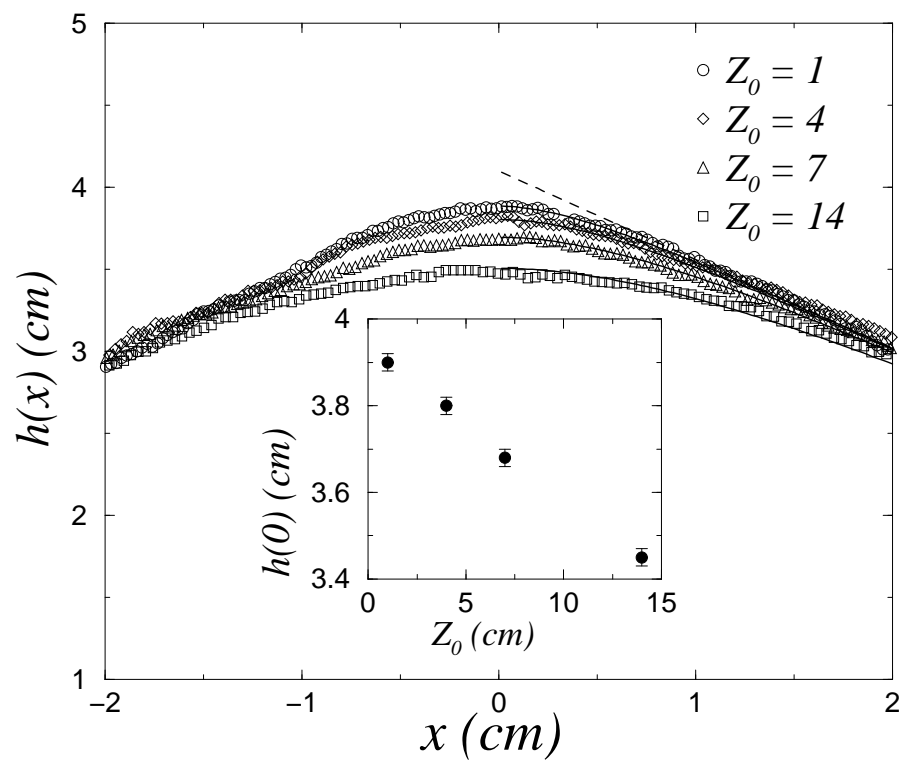

Figure 2: The height profiles of the heaps obtained experimentally with lead beads for different impact energies $Z_{0}$ fitted with Eq. 1. The dashed line has a slope $\theta_{c} \simeq 0.54$. In the inset we show the linear behavior of $h(0)$ with $Z_{0}$. 


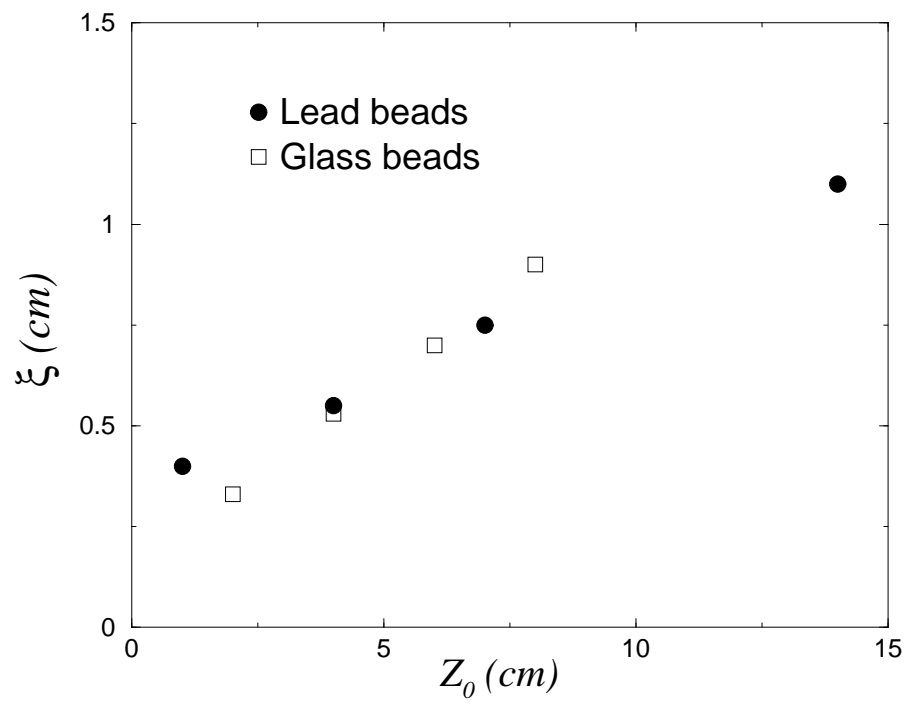

Figure 3: Linear relation between the characteristic lenght $\xi$ and the impact energy $Z_{0}$ for lead and glass beads.

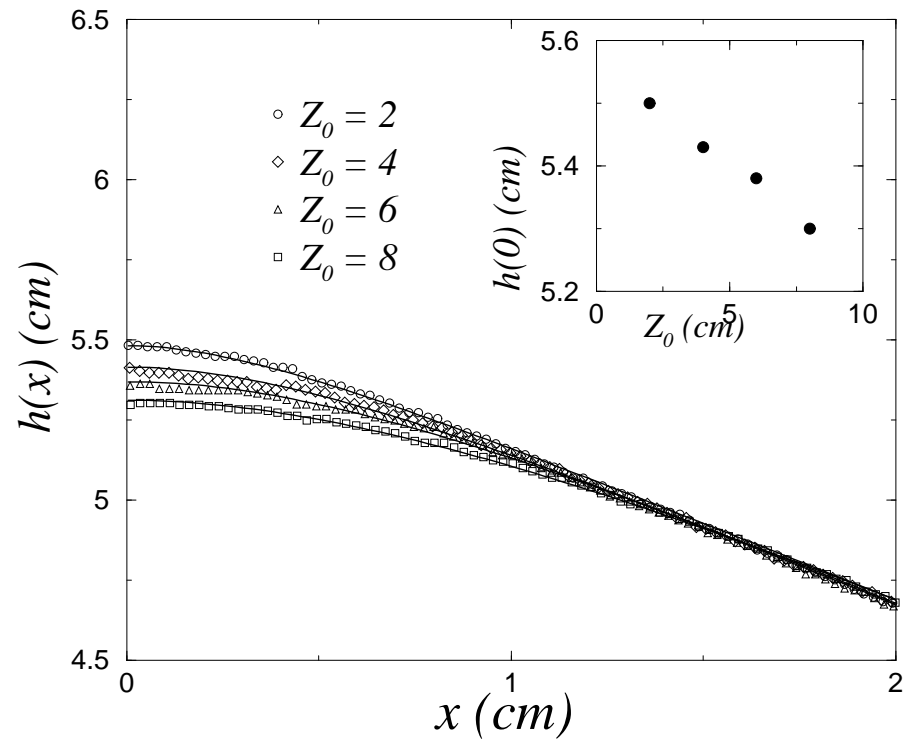

Figure 4: The height profile obtained in the experiment with glass beads, for different impact energies $Z_{0}$ at the lowest available input flux $W=0.32 \mathrm{~g} / \mathrm{s}$. The lines are the fit with Eq. 1. In the inset we show the linear dependence of $h(0)$ with $Z_{0}$. 